\title{
Computer Interfaced Smart A.I Battle Field Tank
}

\author{
Mr. S. Alaguganesan II ME (CSE), Dr. D. Sivakumar M.E., Ph.D \\ Assistant Professor, Department of CSE \\ Kings College of Engineering, Thanjavur, Tamil Nadu, India
}

\begin{abstract}
As the potential for disastrous consequences from threats increases in prevalence, the speed which such cyber threats can occur presents new challenges to understandings of self-defence. This paper first examines the prevention of threats nations could face. It next looks at existing concepts of selfdefence with particular focus on anticipatory and preemptive self-defence, and then moves to a review of the underlying criteria which govern the right to resort to such actions. Highly sophisticated electronic sensors attached to the tank's hull will project images of the surrounding environment back onto the outside of the vehicle enabling it to merge into the landscape and evade attack. The electronic camouflage will enable the vehicle to blend into the surrounding countryside in much the same way that a squid uses ink to help as a disguise. Unlike conventional forms of camouflage, the images on the hull would change in concert with the changing environment always insuring that the vehicle remains disguised.
\end{abstract}

Key Words: Internet of Things (IOT), Sensor, genetic algorithm (GA). Mobile ad-hoc networks (MANETs)

\section{INTRODUCTION}

In this proposed system, the tanker is used to detect the obstacle by capturing the border alert using camera and there is a need of man power to control the machinery. The major disadvantage is the machine is visible and need an man power to control the machine. My aim is to construct an invisible sophisticated tanker. Then introducing a sophisticated tanker which senses the movement in border area without manpower and launches its tube towards the target. Also by using screen and lens over the machine, it has been invisible by adapting towards the environment. In this project, the design a tanker that will be change the color pattern depends on the environment. Every process will be handled and controlled by microcontroller (PIC series).Field instruments such as ultrasonic sensor used to find object movement and range. Depends on the feedback of ultrasonic sensor, the servo motor will turn the launching tube towards the target .Metal sensor used to find the landmine. Selection switch will design the mode to be operated. The proposed system helps to prevent maintenance and human loss by providing invisible mechanism. It senses the obstacles by sensor and allows the launching tube towards the target. In this system the introducing a sophisticated tanker which senses the movement in border area without manpower and launches its tube towards the target. Also by using screen and lens over the machine, it has been invisible by adapting towards the environment.

\section{LITERATURE SURVEY}

\subsection{Transitioning From Federated Avionics} Architectures to Integrated Modular Avionics In this paper identifies considerations for transitioning from a federated avionics architecture to an integrated modular avionics (IMA) architecture. Federated avionics architectures make use of distributed avionics functions that are packaged as self-contained units (LRUs and LRMs).IMA architectures employ a high-integrity, partitioned environment that hosts multiple avionics functions of different criticalities on a shared computing platform. This provides for weight and power savings since computing resources can be used more efficiently. This paper establishes the benefits of transitioning to IMA. To aid in the planning process, the paper also identifies factors to consider before transitioning to IMA. The approach to resource management is identified as the fundamental architectural difference between federated and IMA systems. 


\subsection{Uniform Distribution of Mobile Agents Using Genetic Algorithms for Military Applications In MANETs}

There has been increased research interest in providing uniform distribution of autonomous mobile nodes controlled by active running software agents over an unknown geographical area in mobile ad-hoc networks (MANETs). This problem becomes more challenging under the harsh and bandwidth limited conditions imposed by military applications. In this framework, the software agent running at the application layer for each autonomous mobile node adjusts its direction and speed by using local information from its neighbours. A genetic algorithm (GA) is used by each node to select the speed and direction options among exponentially large number of choices converging towards a uniform distribution.

\subsection{Centralized Vs Distributed Communication Scheme on Switched Ethernet for Embedded Military Applications}

Current military communication network is a generation old and is no longer effective in meeting the emerging requirements imposed by the future embedded military applications. Therefore, a new inter connection system is needed to overcome these limitations. Two new communication networks based upon full duplex switched Ethernet are presented herein in this aim.

\subsection{Smart Military Society: Defining the Characteristics to Score the "Smart" of the Military Services}

Information and Communication Technology (ICT) has been a part of military activities since the invention and massive used of electronic-based systems. Military plays an important role in the emergence of Smart Society today. Using advanced ICT for accomplishing military tasks in advanced countries is a common, but that matter is not the same in military society in developing countries such as Indonesia. Therefore there is a need to define the proper characteristics for a military service so that it can be declared as Smart Military Society. On the other hand, the characteristics of Smart Society itself are not unified yet.

\subsection{Design of Linear Thrusters with a Solid Arc- Shaped Mover Used By Tanker Aircraft}

In this paper, an electric drive linear thrusters is proposed, which is used to control the stretching and shrinking of the oil tube for the tanker aircraft. The major difficulty of this design is to minimize the volume and the weight of the electric actuator. After comparing the advantages and disadvantages of linear induction thrusters with those of permanent magnet linear synchronous thrusters, a curved solid over asynchronous linear thrusters is presented, which reduces the volume and the weight of the device greatly by using the aluminum protection sleeve of the oil tube as the mover and also the secondary.

\section{AIMS AND OBJECTIVES OF THE STUDY}

In this project, the design a tanker that will be change the colour pattern depends on the environment .Every process will be handled and controlled by microcontroller (PIC series).Field instruments such as ultrasonic sensor used to find object movement and range.

Depends on the feedback of ultrasonic sensor, the servo motor will turn the launching tube towards the target. Metal sensor used to find the landmine. Selection switch will design the mode to be operated.

\section{PROPOSED METHODOLOGY}

The proposed system helps to prevent maintenance and human loss by providing invisible mechanism. It senses the obstacles by sensor and allows the launching tube towards the target.

In this system we are introducing a sophisticated tanker which senses the movement in border area without manpower and launches its tube towards the target. Also by using screen and lens over the machine, it has been invisible by adapting towards the environment.

\section{DETECTING METAL SENSOR}

A Metal sensor is a sensor able to detect the presence of nearby objects without any physical contact. A proximity sensor often emits an electromagnetic field or a beam of electromagnetic radiation (infrared, for instance), and looks for changes in the field or return signal. The object being sensed is often referred to as the proximity sensor's target. Different proximity sensor targets demand different sensors. For example, a capacitive or photoelectric sensor might be suitable for a plastic target; an inductive proximity sensor always requires a metal target. The Maximum distance that this sensor can detect is defined "nominal range". Some sensors have adjustments of the nominal range or means to report a graduated detection distance. Proximity sensors can have a high 
reliability and long functional life because of the absence of mechanical parts and lack of physical contact between sensor and the sensed object. Metal sensors are commonly used on smart phones to detect (and skip) accidental touch screen taps when held to the ear during a call. They are also used in machine vibration monitoring to measure the variation in distance between a shaft and its support bearing. This is common in large steam turbines, compressors, and motors that use sleeve-type bearings.

\section{DETECTING INFRARED SENSOR}

Infrared (IR) light is electromagnetic radiation with a wavelength longer than that of visible light, measured from the nominal edge of visible red light at 0.74 micrometers $(\mu \mathrm{m})$, and extending conventionally to $300 \mu \mathrm{m}$. These wavelengths correspond to a frequency range of approximately 1 to $400 \mathrm{THz}$, and include most of the thermal radiation emitted by objects near room temperature. Microscopically, IR light is typically emitted or absorbed by molecules when they change their rotational-vibration movements. Infrared light is used in industrial, scientific, and medical applications. Night-vision devices using infrared illumination allow people or animals to be observed without the observer being detected. In astronomy, imaging at infrared wavelengths allows observation of objects obscured by interstellar dust. Infrared imaging cameras are used to detect heat loss in insulated systems, observe changing blood flow in the skin, and overheating of electrical apparatus.

An infrared sensor is an electronic device that emits and/or detects infrared radiation in order to sense some aspect of its surroundings. Infrared sensors can measure the heat of an object, as well as detect motion. Many of these types of sensors only measure infrared radiation, rather than emitting it, and thus are known as passive infrared (PIR) sensors.All objects emit some form of thermal radiation, usually in the infrared spectrum. This radiation is invisible to our eyes, but can be detected by an infrared sensor that accepts and interprets it. In a typical infrared sensor like a motion detector.

Most motion detectors are fitted with a special type of lens, called a Fresnel lens, on the sensor face. A set of these lenses on a motion detector can focus light from many directions, giving the sensor a view of the whole area. Instead of Fresnel lenses, some motion detectors are fitted with small parabolic mirrors which serve the same purpose.

\section{IMPLEMENTATION RESULTS}

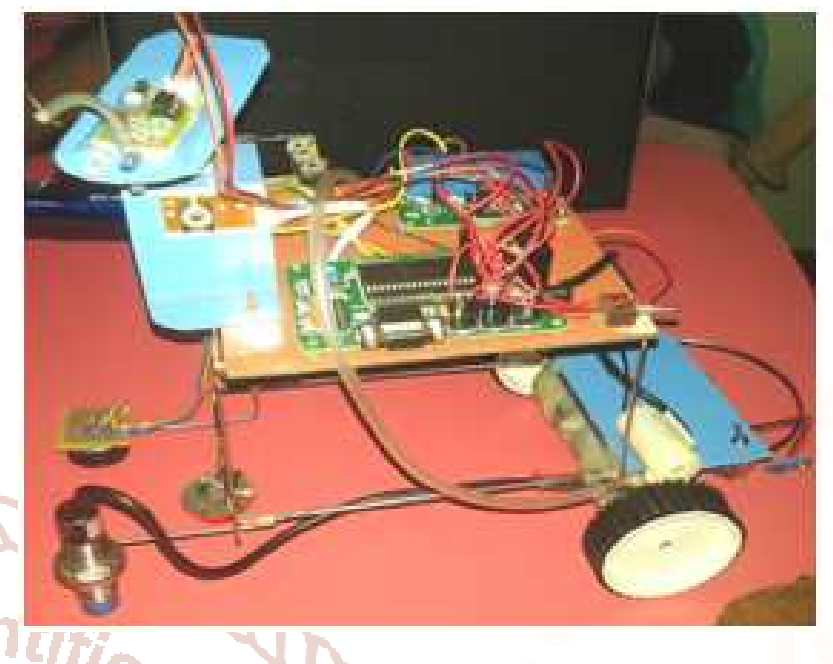

\section{CONCLUSION}

The intent was to provide on overall idea of the activities and also some details on specifications and requirements relevant to military applications. In the military, there are various applications that call for much higher power than is necessary in regular commercial vehicles, and the environment of the application can be very harsh in terms of temperature and vibration. Some of the important differences between military and regular commercial applications were noted. To meet the army's needs, both robust and high efficiency devices are important.

\section{FUTURE ENHANCEMENT}

To introduce a new tanker system that will be invisible, accurate, transparent, fast and will ensure a safe military system. Our proposed system has covered all of these issues successfully. Moreover this system will provide automated border alert system. The system should also verify that the electronic system has not been compromised. This includes validation of physical machines for consistency and for material weakness. In this project, design and implementation of invisible border alert system in military system were presented .A prototype was successfully developed and tested to establish the proof of concept.

\section{REFERENCES}

1. Van Drongelen, B. Roszek, E. S. M. HilbersModderman,M. Kallewaard, And C. Wassenaar, Wheelchair Incidents, November 2002, Accessed February, 2010. 
2. Frank, J. Ward, N. Orwell, C. Mccullagh, And M. Belcher, Introduction Of A New NHS ElectricPowered Indoor/Outdoor Chair (EPIOC) Service: Benefits, Risks And implications For Prescribers, Clinical Reha-Bilitation, No. 14, Pp. 665- 673, 2000.

3. R. C. Simpson, E. F. Lopresti, And R. A. Cooper, How Many People Would Benefit From A Smart Wheelchair? Journal Of Rehabilitation research And Development, Vol. 45,No. 1,Pp. 53-71,2008

4. T. Carlson And Y. Demiris, Collaborative Control For A Robotic Wheelchair:Evaluation Of Performance, Attention, And Workload, IEEE Transactions On Systems, Man, And Cybernetics, Part B: Cybernetics, Vol.42, No.3, Pp.876888,2012 .

5. B. Rebsamen, C. Guan, H. Zhang, C. Wang, C. Teo, M.Ang, And E. Burdet, A Brain Controlled Wheelchair To Navigate In Familiar Environments, IEEE Transactions On Neural Systems And Rehabilitation Engineering, Vol.18, No. 6, Pp. 590-598, Dec. 2010.

6. Iturrate, J. Antelis, A. Kubler," And J. Minguez, A Noninvasive Brain-Actuated Wheelchair Based
On A P300 Neuro physiological Protocol And Automated Navigation, IEEE Transactions On Robotics, Vol. 25, No. 3, Pp. 614-627, June 2009.

7. J. D. R. Millan, F. Galan, D. Vanhooydonck, E. Lew, J. Philips, And M. Nuttin , Asynchronous Non-Invasive Brain-Actuated Control Of An Intelligent heelchair, In Proc. 31st Annual Int. Conf. IEEE Eng. Med. Biol. Soc., 2009, Pp. 3361-3364.

8. J. D. R. Millan, F. Renkens, J. Mourino, And W. Gerstner, Noninvasive Brain-Actuated Control Of A Mobile Robot By Human EEG, IEEE Trans Biomed Eng, Vol. 51, No. 6, Pp. 10261033, 2004.

9. F. Galan P. W. Ferrez, F. Oliva, J. Guardia, and J. D. R. Millan Feature Extraction for Multi-Class BCI Using Canonical Variates Analysis, In IEEE IntSymp ISP Intelligent Signal Processing, 2007.

10. J. D. R. Millan, P. W. Ferrez, F. Gal An, E. Lew, And R. Chavarriaga, Non Invasive Brain-Machine Interaction, Int $\mathbf{J}$ Pattern Recognition And Artificial Intelligence, Vol. 22, No. 5, Pp. 959972, 2008. 